\title{
Drug-Induced Sleep Endoscopy: A Guide for Treatment Selection
}

\author{
Jin Youp Kim, MD¹, Seung Cheol Han, MD¹, Hyun Jung Lim, MD¹, Hyun Jik Kim, MD, PhD¹, \\ Jeong-Whun Kim, MD, PhD¹, Chae-Seo Rhee, MD, $\mathrm{PhD}^{1,2,3,4}$ \\ ${ }^{1}$ Department of Otorhinolaryngology-Head and Neck Surgery, ${ }^{2}$ Graduate School of Immunology, Seoul National University College of Medicine, Seoul, \\ Korea \\ ${ }^{3}$ Institute of Allergy and Clinical Immunology, ${ }^{4}$ Sensory Organ Research Institute, Medical Research Center, Seoul National University College of Medicine, \\ Seoul, Korea
}

\author{
Received: May 5, 2020 \\ Revised: May 25, 2020 \\ Accepted: May 27, 2020 \\ Correspondence \\ Chae-Seo Rhee, MD, PhD \\ Department of Otorhinolaryngology-Head \\ and Neck Surgery, \\ Seoul National University Hospital, \\ 101 Daehak-ro, Jongno-gu, \\ Seoul 03080, Korea \\ Tel +82-2-2072-3991 \\ Fax +82-2-766-2447 \\ E-mail csrhee@snu.ac.kr \\ ORCID \\ Jin Youp Kim \\ https://orcid.org/0000-0003-2687-6919 \\ Seung Cheol Han \\ https://orcid.org/0000-0003-1981-9334 \\ Hyun Jung Lim \\ https://orcid.org/0000-0003-0064-4669 \\ Hyun Jik Kim \\ https://orcid.org/0000-0001-8631-928X \\ Jeong-Whun Kim \\ https://orcid.org/0000-0003-4858-3316 \\ Chae-Seo Rhee \\ https://orcid.org/0000-0002-1361-8585
}

(c) This is an Open Access article distributed under the terms of the Creative Commons Attribution Non-Commercial License (https://creativecommons.org/licenses/by-nc/4.0) which permits unrestricted non-commercial use, distribution, and reproduction in any medium, provided the original work is properly cited.
Identifying the sites, severity, and pattern of upper airway obstruction in obstructive sleep apnea is crucial for determining the treatment options, particularly the surgical plan. Although early methods of assessment, such as Muller's maneuver, computed tomography, and magnetic resonance imaging in awake patients have been utilized to assess obstructive sites, the physiologic and anatomical differences between awake and sleeping patients showed a limited ability of those methods as diagnostic tools. Recently, drug-induced sleep endoscopy (DISE) was introduced as a useful diagnostic and evaluation tool to identify dynamic upper airway collapse during sedation that simulates natural sleep. In this article, we review all aspects of DISE, including the technique, evaluation methods, and clinical application.

Sleep Med Res 2020;11(1):1-6

Key Words Sleep apnea, Obstructive, Drug-induced sleep endoscopy, Sedation, Snoring.

\section{INTRODUCTION}

Obstructive sleep apnea (OSA) is a common sleep disorder, characterized by recurrent collapse of the upper airways during sleep. Because a wide spectrum of pharyngeal dysfunction is found in OSA, evaluation of the obstruction site and its severity in patients with OSA is extremely important for selecting the treatment method. There are four treatment options for OSA, including positive airway pressure (PAP), oral devices, surgery, and lifestyle modifications. To date, PAP is thought to be the most effective treatment but shows the problem of compliance. Therefore, surgery and oral devices play specific roles for some patients with OSA. Although the expected success rate of surgery is relatively lower than that of PAP, it is an effective option for patients who have obvious anatomic problems or want to be treated with surgery at once. In those patients, it is mandatory to evaluate the obstructive level of the airways during sleep. Several techniques for evaluation of the airways have been proposed, including clinical examination with Muller's maneuver, cephalometry, computed tomography (CT), magnetic resonance imaging (MRI), acoustic reflectometry, sleep videofluoroscopy (SVF), and drug-induced sleep endoscopy (DISE). Although early methods of evaluation, such as Muller's maneuver, CT, and MRI in awake patients were utilized to assess the obstructive site, the physiologic and anatomical differences between awake and sleeping patients showed a limited ability as evaluation tools. SVF and DISE can be used under sedation mimicking sleep. SVF has several advantages: dynamic sleep study, performed in the supine position, and visualization of the both the head and neck, including bony and soft structures and upper airways together. However, it has the limitations of radiation exposure, superimposition of structures, and two-dimensional view. Recently, most OSA surgeons utilized DISE to determine the surgical options, particularly using general anesthesia, although DISE has disadvantages related to identifying the entire air- 
ways together.

As observation of the obstructive site during sleep is necessary for proper evaluation, Croft and Pringle [1] first suggested "sleep nasoendoscopy" to assess OSA patients in 1991. As a pharmacological agent is required to sedate patients, Kerzirian and Hohenhorst [2] changed the nomenclature to "drug-induced sleep endoscopy." In contrast to the previous procedures that commonly provide two-dimensional evaluations in the upright sitting and awake state, DISE provides a three-dimensional assessment of the upper airways during unconscious sedation that simulates natural sleep.

\section{TECHNIQUE}

\section{Preparation and Patient Positioning}

Eating should be restricted for patients before the procedure to prevent aspiration during the procedure. Anticholinergic agents, such as atropine and glycopyrrolate, can be administrated $30 \mathrm{~min}$ before the procedure to decrease salivary secretion, which leads to a better window of the upper airway and reduces coughing caused by aspiration. A local decongestant was commonly applied to the unilateral or bilateral nasal airways. The amount of local anesthesia should be minimalized to avoid anesthetic extension to the pharynx and affecting the genioglossus [3]. Endoscopic examination should be performed before administration of the sedative to verify the anesthetic state.

\section{Sedative Agents}

Propofol (2-6-diisopropylphenol) and midazolam (benzodiazepine) are commonly used as sedative agents during DISE. Dexmedetomidine (alpha-2 adrenergic receptor agonist) is also utilized. Propofol is a hypnotic agent, but its mechanism of its action remains unknown; it is believed that gamma-amino-butyric acid (GABA) circuits are associated with the mechanism of action of propofol. It can reproduce slow waves on electroencephalogram, which resembles the waves of non-rapid eye move- ment (NREM) sleep. Previous studies demonstrated that pharyngeal pressures under propofol sedation were equal to those in natural NREM sleep [4,5]. Midazolam is a short-acting benzodiazepine that increases the opening frequency of the GABA-A receptor. Theoretically, it produces greater relaxation of the pharyngeal muscle compared to propofol. However, studies measuring the pharyngeal pressure showed that pharyngeal pressures under midazolam sedation also corresponded to those of NREM natural sleep [6,7]. Table 1 describes the advantages, disadvantages, and doses of propofol and midazolam.

\section{Setting and Monitoring}

DISE is safely performed in an operating room or a procedure suite but can also be performed in an outpatient setting with the basic resuscitation equipment (e.g., supplemental oxygen) and available personnel for safe administration of the sedative. Comfortable room temperature, a quiet environment, and dim lights are helpful to simulate natural sleep. The oxygen saturation, heart rate, and blood pressure must be monitored during the procedure, and supplemental oxygen should be prepared in case of emergency. Sedation depth can be assessed with the loss of consciousness, onset of snoring, and disordered breathing events. The bispectral index score (BIS) provides a quantified sedation depth. The recommended target BIS range during DISE is 50 to 70 [8]. A previous study demonstrated more upper airway collapse in deep sedation (BIS of 50 to 60) compared to light sedation (BIS of 65-75), which suggested that a higher level of sedation may cause a greater loss of muscle tone [9]. Therefore, a constant sedation depth should be set with a definite protocol for the individual sleep laboratory.

\section{Evaluation of the Upper Airways in DISE}

Various classifications have been reported to describe DISE findings. Although there is no consensus, the VOTE classification is the most widely used because it integrates the four major structures that contribute to upper airway obstruction in most patients: velum, oropharyngeal lateral walls, tongue base, and

Table 1. Sedative agents of drug-induced sleep endoscopy

\begin{tabular}{|c|c|c|c|}
\hline & Advantage & Disadvantage & Dose \\
\hline \multirow[t]{8}{*}{ Propofol } & Quick and safe manageable & Technique dependent (need & TCI \\
\hline & Less muscle relaxation & pump or TCI) & Starting $1.5-3.0 \mu \mathrm{g} / \mathrm{mL}$ \\
\hline & More closely mimics natural sleep & & If required increase with rate of $1.5-3.0 \mu \mathrm{g} / \mathrm{mL}$ \\
\hline & Easier control of titration & & Pump \\
\hline & & & Delivering dose, 50-100 mL/hour \\
\hline & & & Bolus \\
\hline & & & Starting $30-50$ or $1 \mathrm{mg} / \mathrm{kg}$ \\
\hline & & & If required increase with rate of $10-20 \mu \mathrm{g} / \mathrm{mL}$ \\
\hline \multirow[t]{3}{*}{ Midazolam } & Longer and more stable examination & More difficult to handle in case & Bolus \\
\hline & window & of overdosing & Starting: $0.03 \mathrm{mg} / \mathrm{kg}$ \\
\hline & Antidote available & Longer hospital stay & If required increased with $0.015-0.030 \mathrm{mg} / \mathrm{kg}$ \\
\hline
\end{tabular}

TCI: target-controlled infusion. 

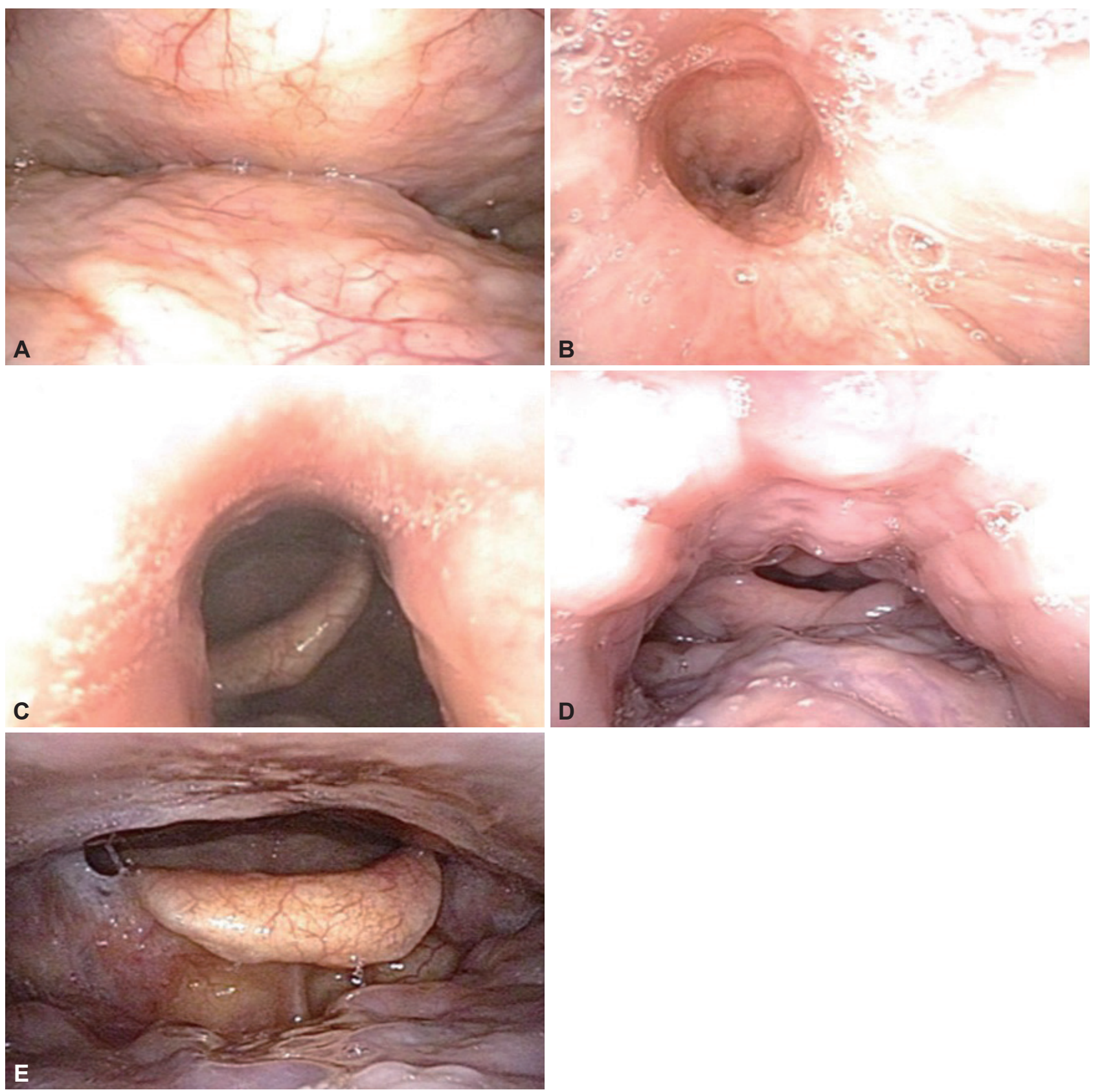

Fig. 1. VOTE classification. A: Velum obstruction in the anteroposterior configuration. B: Velum obstruction in the concentric configuration. C: Oropharyngeal lateral wall collapse. D: Tongue-related obstruction. E: Epiglottic collapse in the anteroposterior configuration. VOTE: velum, oropharyngeal lateral walls, tongue base, and epiglottis.

epiglottis [10]. Fig. 1 shows the four sites of obstruction in the VOTE classification. In addition, the NOHL classification, which includes the nose, oropharynx, hypopharynx, and larynx, is used [11]. Table 2 summarizes these two classification systems. VOTE and NOHL classifications share similarities in most parameters to identify the site, degree, and pattern of obstruction of the upper airways. The NOHL classification may be more advantageous because it provides an additional level of nasal obstruction. In contrast, a previous study comparing VOTE and NOHL classification showed that the VOTE classification was more compre- hensive in the assessment of the pharynx and epiglottis [12].

\section{CLINICAL APPLICATION}

The clinical purpose of DISE is to guide treatment selection based on the association between the findings in DISE and outcomes of various treatment modalities. Multiple studies have examined the DISE as a diagnostic tool that aids in treatment planning [13-16]. A study described that DISE may change the 
treatment recommendations in $78 \%$ of subjects [15]. Since it does not evaluate the severity of OSA, complete polysomnography or portable monitoring should be performed before DISE. Some aspects of the upper airway anatomy are better observed in the awake state; therefore, a complete awake upper airway examination is also important to aid in treatment selection. DISE is indicated for OSA patients and simple snorers when additional information provided by DISE is considered important. For example, DISE is not necessary for all patients treated with continuous positive airway pressure (CPAP). However, if the patient does not tolerate CPAP, DISE can be utilized to determine the possible effectiveness of alternative treatments including upper airway surgery and oral devices. DISE cannot be safely performed in patients with American Society of Anesthesiologists class 4, pregnancy, or allergies to sedative agents [17]. A high apnea-hypopnea index (AHI) score is not considered as a contraindication for DISE [17].

\section{Upper Airway Surgery}

In a previous systemic review, the plan for OSA surgery was changed in $50.2 \%$ of patients after performing DISE, and the changes were mainly related to obstructions in the hypopharynx or larynx compared to awake endoscopy [18]. However, the changes did not automatically lead to favorable surgical outcomes [18]. In a previous study, complete collapse of the lateral pharyngeal wall showed a strong association with the severity of OSA [19]. In addition, improved stability of the lateral pharyngeal wall observed in DISE showed a direct correlation with improved AHI after maxillomandibular advancement procedures [20]. Concentric palatal obstruction or hypopharyngeal-related obstruction was related to worse outcomes after uvulopalatopharyngoplasty $[21,22]$. Moreover, tongue-related obstruction or complete concentric collapse of the velum was related to worse outcomes in patients who underwent one or more surgical procedures for OSA [23]. Therefore, patients with complete concentric collapse of the pharynx should be treated with PAP rather than with OSA surgery. However, if a patient refuses to undergo CPAP and wants an operation, OSA surgery with tonsillectomy and soft palate surgery, which can sustain the lateral wall of the pharynx, such as suspension lateral pharyngoplasty, relocation pharyngoplasty, expansion sphincter pharyngoplasty, zpharyngoplasty, and barbed reposition pharyngoplasty can be performed as an alternative treatment. DISE may show obstruction of the laryngeal inlet caused by posterior retraction of the epiglottis, and surgical laser wedge resection can be performed to open the laryngeal inlet [24]. However, as a recent European position paper on DISE indicated, further investigations are required to evaluate in detail whether or not certain DISE findings are related to treatment outcomes [25]. Table 3 summarizes the summarized the recommendation of treatment options from

Table 2. Summary of drug-induced sleep endoscopy classification system

\begin{tabular}{|c|c|c|c|c|c|}
\hline & Structure & Degree of obstruction & & Configuration & \\
\hline \multirow[t]{6}{*}{ VOTE classification } & Velum & 0: no obstruction & Anteroposterior & Lateral & Concentric \\
\hline & Oropharynx lateral walls & 1: partial obstruction & Not visualized & Lateral & Not visualized \\
\hline & Tongue base & 2: complete obstruction & & & \\
\hline & Epiglottis & & Anteroposterior & Not visualized & Not visualized \\
\hline & & & Anteroposterior & Lateral & Not visualized \\
\hline & Site of obstruction & Grade & & Configuration & \\
\hline \multirow[t]{5}{*}{ NOHL classification } & Nose & 0-25\%: 1 & - & - & - \\
\hline & Oropharynx & $25-50 \%: 2$ & Anteroposterior & Lateral & Concentric \\
\hline & Hypopharynx & $50-75 \%: 3$ & Anteroposterior & Lateral & Concentric \\
\hline & & 75-100\%: 4 & - & - & - \\
\hline & Larynx (supraglottis, glottis) & Positive or negative obstruction & & & \\
\hline
\end{tabular}

Table 3. Recommendation of treatment options according to drug-induced sleep endoscopy findings

\begin{tabular}{lll}
\hline \multicolumn{1}{c}{ Obstruction sites } & \multicolumn{1}{c}{ Configuration } & \multicolumn{1}{c}{ Recommended treatment option } \\
\hline Velum or oropharynx & Anteroposterior obstruction & Soft palate surgery (e.g., UPPP, uvulopalatal flap) \\
& Anteroposterior obstruction relieved by jaw thrust maneuver & Soft palate surgery (e.g., UPPP, uvulopalatal flap) \\
& & or MAD \\
Tongue base & Lateral wall collapse/complete concentric collapse & PAP/surgery* \\
& Upper tongue & PAP or MAD \\
Epiglottis & Lower tongue (lingual tonsil) & Lingual tonsillectomy using coblator or robot \\
\hline
\end{tabular}

*If a patient want the operation, surgery with tonsillectomy and suspension lateral pharyngoplasty can be an alternative.

UPPP: uvulopalatopharyngoplasty, PAP: positive airway pressure, MAD: mandibular advancement device. 
previous studies according to DISE findings. The association between DISE findings and outcomes of OSA surgery may help clinicians select proper candidates for OSA surgery. A previous study evaluated the effectiveness of DISE to determine the surgical plan for single- or multi-level surgeries and upper airway stimulation. They found that the subjects who underwent DISE had a higher success rate for OSA surgery than those who did not $(84 \%$ vs. $52 \%, \mathrm{p}<0.001)$ [26]. In summary, the role of DISE for OSA surgery is to identify localization of obstruction sites, eliminate unnecessary procedures, and improve clinical outcomes.

\section{Mandibular Advanced Device}

Mandibular advanced device (MAD) is an effective treatment option for mild to moderate OSA. The jaw-thrust maneuver during DISE is used to predict the effect of MAD. Patients with a substantial improvement in upper airway patency during the jaw-thrust maneuver had better outcomes with MAD [27]. In addition, $97 \%$ of the patients who showed increased airway dimensions with MAD during DISE achieved favorable treatment outcomes with MAD [16]. Therefore, MAD may be indicated for patients with improvement in upper airway patency with the jaw-thrust maneuver or MAD during DISE.

\section{Limitation of DISE}

There are some limitations of DISE as an evaluation tool for OSA. First, in contrast to polysomnography, DISE does not reflect the information for the entire night. Therefore, the findings in DISE could be a snapshot of the sleep events during the night. Second, unlike SVF, the whole airway cannot be seen at once in DISE. Therefore, DISE could be used complementary to SVF [28]. Lastly, standardization of setting in sedation and evaluation system is limited. Although some hospital-based standardization of sedative agents and the evaluation system was established, considering that DISE reflects the subjective view of the operator through the endoscope, general standardization is necessary.

\section{SUMMARY}

Identifying the obstruction sites of the upper airways, with their severity and pattern, in OSA is crucial for determining the treatment plan. DISE is a safe and useful tool to assess dynamic upper airway collapse before surgery. DISE findings are helpful to determine the best treatment option for OSA patients. Although DISE is commonly utilized to aid surgical decision making, it can also assist the optimization of medical therapy, such as MAD.

\section{Acknowledgments}

None.

\section{Conflicts of Interest}

The authors have no financial conflicts of interest.

\section{Authors' Contribution}

Conceptualization: Rhee CS. Data curation: Kim JY, Han SC. Investigation: Rhee CS, Kim JW, Kim HJ. Methodology: Kim JY, Lim HJ, Han SC. Project administration: Rhee CS. Resources: Rhee CS, Kim JW, Kim HJ. Supervision: Rhee CS. Validation: Rhee CS, Kim JW, Kim HJ. Visualization: Kim JY, Lim HJ. Writing-original draft: Kim JY, Rhee CS. Writing_review \& editing: Rhee CS, Kim JW, Kim HJ.

\section{REFERENCES}

1. Croft CB, Pringle M. Sleep nasendoscopy: a technique of assessment in snoring and obstructive sleep apnoea. Clin Otolaryngol Allied Sci 1991;16:504-9.

2. Charakorn N, Kezirian EJ. Drug-induced sleep endoscopy. Otolaryngol Clin North Am 2016;49:1359-72.

3. Fogel RB, Malhotra A, Shea SA, Edwards JK, White DP. Reduced genioglossal activity with upper airway anesthesia in awake patients with OSA. J Appl Physiol (1985) 2000;88:1346-54.

4. Hillman DR, Walsh JH, Maddison KJ, Platt PR, Kirkness JP, Noffsinger WJ, et al. Evolution of changes in upper airway collapsibility during slow induction of anesthesia with propofol. Anesthesiology 2009;111: 63-71.

5. Hoshino Y, Ayuse T, Kurata S, Ayuse T, Schneider H, Kirkness JP, et al. The compensatory responses to upper airway obstruction in normal subjects under propofol anesthesia. Respir Physiol Neurobiol 2009;166: 24-31.

6. Llatas MC, Porras GA, González MTC, Sanbartolomé AR, Bayarri PG, Gómez-Pajares F, et al. Drug-induced sleep endoscopy: a two drug comparison and simultaneous polysomnography. Eur Arch Otorhinolaryngol 2014;271:181-7.

7. Genta PR, Eckert DJ, Gregorio MG, Danzi NJ, Moriya HT, Malhotra A, et al. Critical closing pressure during midazolam-induced sleep. $J$ Appl Physiol (1985) 2011;111:1315-22.

8. De Vito A, Carrasco Llatas M, Vanni A, Bosi M, Braghiroli A, Campanini A, et al. European position paper on drug-induced sedation endoscopy (DISE). Sleep Breath 2014;18:453-65.

9. Amos JM, Durr ML, Nardone HC, Baldassari CM, Duggins A, Ishman SL. Systematic review of drug-induced sleep endoscopy scoring systems. Otolaryngol Head Neck Surg 2018;158:240-8.

10. Kezirian EJ, Hohenhorst W, de Vries N. Drug-induced sleep endoscopy: the VOTE classification. Eur Arch Otorhinolaryngol 2011;268: 1233-6.

11. Vicini C, De Vito A, Benazzo M, Frassineti S, Campanini A, Frasconi $\mathrm{P}$, et al. The nose oropharynx hypopharynx and larynx (NOHL) classification: a new system of diagnostic standardized examination for OSAHS patients. Eur Arch Otorhinolaryngol 2012;269:1297-300.

12. da Cunha Viana A Jr, Mendes DL, de Andrade Lemes LN, Thuler LCS, Neves DD, de Araújo-Melo MH. Drug-induced sleep endoscopy in the obstructive sleep apnea: comparison between NOHL and VOTE classifications. Eur Arch Otorhinolaryngol 2017;274:627-35.

13. Aktas O, Erdur O, Cirik AA, Kayhan FT. The role of drug-induced sleep endoscopy in surgical planning for obstructive sleep apnea syndrome. Eur Arch Otorhinolaryngol 2015;272:2039-43.

14. Vanderveken OM, Vroegop AV, Van de Heyning PH, Braem MJ. Druginduced sleep endoscopy completed with a simulation bite approach for the prediction of the outcome of treatment of obstructive sleep apnea with mandibular repositioning appliances. Oper Tech Otolayngol Head Neck Surg 2011;22:175-82.

15. Eichler C, Sommer JU, Stuck BA, Hörmann K, Maurer JT. Does druginduced sleep endoscopy change the treatment concept of patients with snoring and obstructive sleep apnea? Sleep Breath 2013;17:63-8.

16. Johal A, Battagel JM, Kotecha BT. Sleep nasendoscopy: a diagnostic tool for predicting treatment success with mandibular advancement splints in obstructive sleep apnoea. Eur J Orthod 2005;27:607-14.

17. Carrasco-Llatas M, Matarredona-Quiles S, De Vito A, Chong KB, Vi- 
cini C. Drug-induced sleep endoscopy: technique, indications, tips and pitfalls. Healthcare (Basel) 2019;7:93.

18. Certal VF, Pratas R, Guimarães L, Lugo R, Tsou Y, Camacho M, et al. Awake examination versus DISE for surgical decision making in patients with OSA: a systematic review. Laryngoscope 2016;126:768-74.

19. Lan MC, Liu SY, Lan MY, Modi R, Capasso R. Lateral pharyngeal wall collapse associated with hypoxemia in obstructive sleep apnea. Laryngoscope 2015;125:2408-12.

20. Liu SY, Huon LK, Powell NB, Riley R, Cho HG, Torre C, et al. Lateral pharyngeal wall tension after maxillomandibular advancement for obstructive sleep apnea is a marker for surgical success: observations from drug-induced sleep endoscopy. J Oral Maxillofac Surg 2015;73:1575-82.

21. Iwanaga K, Hasegawa K, Shibata N, Kawakatsu K, Akita Y, Suzuki K, et al. Endoscopic examination of obstructive sleep apnea syndrome patients during drug-induced sleep. Acta Otolaryngol Suppl 2003; (550):36-40.

22. Hessel NS, Vries N. Increase of the apnoea-hypopnoea index after uvulopalatopharyngoplasty: analysis of failure. Clin Otolaryngol Allied Sci 2004;29:682-5.
23. Koutsourelakis I, Safiruddin F, Ravesloot M, Zakynthinos S, de Vries N. Surgery for obstructive sleep apnea: sleep endoscopy determinants of outcome. Laryngoscope 2012;122:2587-91.

24. Kotecha B, De Vito A. Drug induced sleep endoscopy: its role in evaluation of the upper airway obstruction and patient selection for surgical and non-surgical treatment. J Thorac Dis 2018;10(Suppl 1):S40-7.

25. De Vito A, Carrasco Llatas M, Ravesloot MJ, Kotecha B, De Vries N, Hamans E, et al. European position paper on drug-induced sleep endoscopy: 2017 update. Clin Otolaryngol 2018;43:1541-52.

26. Huntley C, Chou D, Doghramji K, Boon M. Preoperative drug induced sleep endoscopy improves the surgical approach to treatment of obstructive sleep apnea. Ann Otol Rhinol Laryngol 2017;126:478-82.

27. Johal A, Hector MP, Battagel JM, Kotecha BT. Impact of sleep nasendoscopy on the outcome of mandibular advancement splint therapy in subjects with sleep-related breathing disorders. J Laryngol Otol 2007; 121:668-75

28. Hong SN, Won TB, Kim JW, Lee CH, Rhee CS. Upper airway evaluation in patients with obstructive sleep apnea. Sleep Med Res 2016;7:1-9. 